\title{
Quantification of tumour vascularity in squamous cell carcinoma of the tongue using CARD amplification, a systematic sampling technique, and true colour image analysis
}

\author{
Egied J.M. Hannen a,b,*, \\ Jeroen A.W.M. van der Laak ${ }^{\mathrm{a}}$, \\ Harold M.J. Kerstens ${ }^{\text {a }}$, Vincent M.J.I. Cuijpers ${ }^{\text {a }}$, \\ Antonius G.J.M. Hanselaar ${ }^{\text {a }}$, Johannes J. Manni ${ }^{\mathrm{c}}$ \\ and Peter C.M. de Wilde ${ }^{\mathrm{a}}$ \\ ${ }^{a}$ Department of Pathology, University Medical Centre \\ Nijmegen, The Netherlands \\ ${ }^{\mathrm{b}}$ Department of Cranio and Maxillofacial Surgery, \\ University Hospital Maastricht, The Netherlands \\ ${ }^{\mathrm{c}}$ Department of Otorhinolaryngology, Head and Neck \\ Surgery, University Hospital Maastricht, \\ The Netherlands
}

Received 25 August 2000

Accepted 27 March 2001

The aims of this study of head and neck tissue samples were to develop an immunohistochemical protocol based on the catalysed reporter deposition (CARD) technique to enhance staining results for use in automated true colour image analysis, to assess the reproducibility of systematic tissue sampling in the angiogenic hot spot selection, and quantification of microvessel density (MVD) and other vessel characteristics. The latter data were compared between six metastasised tongue squamous cell carcinomas, vs. four non-metastasised. In comparison to the standard immunohistochemical protocol with anti-CD34 antibodies, CARD amplification resulted in both more intensely stained and larger numbers of vessels. Averaging the 10 most vascularised fields of the 40 to 60 systematically sampled fields in a tissue section resulted in an overall acceptable interobserver reproducibility for most assessed vessel parameters $(r \geqslant 0.76$ and $p \leqslant 0.01)$.

\footnotetext{
${ }^{*}$ Corresponding author: E.J.M. Hannen, DMD, MD, Department of Cranio and Maxillofacial Surgery, University Hospital Maastricht, PO Box 5800, 6202 AZ Maastricht, The Netherlands. Tel.: +31 43387 2010; Fax: +31 43387 2020, E-mail: ehan@mkg.azm.nl.
}

The percentage vessels with diameter $<5 \mu \mathrm{m}$ was significantly higher in the non-metastasised tongue carcinomas $(p=0.02)$. However, for a number of tumours the effect of tissue sampling was significant.

We conclude that CARD amplification is needed for reliable segmentation of vessels by image analysis systems, and that tumour heterogeneity is a limiting factor for all procedures in which tumour vascularity is assessed in a single tissue section. htm.

Figures on http://www.esacp.org/acp/2001/22-4/hannen.

\section{Introduction}

The angiogenesis-inducing capacity of malignant tumours is regarded as an important factor influencing tumour growth and clinico-biological behaviour [6]. Correlations between high microvessel density (MVD) and the occurrence of metastases have been reported for several types of malignancies $[6,14,27]$, including squamous cell carcinomas of the head and neck $[8$, $9,16,21,22,29]$. Measurement of MVD is mostly performed by manual counting of vascular profiles in tissue sections in which blood vessels are visualised by immunohistochemical procedures. Most widely used is a protocol described by Weidner et al. [27], in which vessels are manually counted in the so-called "angiogenic hot spot". The hot spot is defined as the area of a tumour with the highest degree of vascularisation and MVD is defined as the number of microvessels in the hot spot [27] or as the mean value of MVD assessed over a small number of the most vascularised areas of the tumour [14]. The limitations of this procedure are discussed in a recent manuscript on an international consensus on the methodology and evaluation criteria of angiogenesis quantification [25]. 
In a recent study, it was shown that true colour image analysis is a good alternative to manual counting, to quantify tumour vascularity in the hot spot [24]. In that study on human melanoma xenografts, the whole tumour in a tissue section was completely sampled with non-overlapping, consecutive standardised fields, and subsequently the average vessel density over the three highest vascularised fields was used to define the automated hot-spot MVD. This sampling procedure warranted that the highest vascularised fields were almost always found by independent observers, but it is a very time-consuming method, especially for large tumours. A tissue sampling technique not yet well explored in studies on angiogenesis is the procedure of systematic sampling [4] that is often used in stereology $[11,26]$. To the best of our knowledge, only few published studies on microvessel counting used a systematic sampling procedure $[5,13,28]$, and it is not considered in the aforementioned consensus report [25].

In a preliminary study on angiogenesis in squamous cell carcinomas (SCCs) of the tongue, we encountered the problem that the intensity of the immunohistochemically visualised vessels was sometimes very low. In some cases, the intensity of the immunohistochemical signal was so weak that the vessels were hardly visible. Furthermore, large variations existed between tumours of different patients (i.e., inter-tumour variation) and within the same tumour (i.e., intra-tumour variation). Especially the intertumour variations were considerable, even when a standardised immunohistochemical procedure was used. The results of recognition of vessel profiles by an image analysis system are mainly determined by the quality of the immunohistochemical procedure [24]. Conventional immunohistochemical techniques, including those using antigenretrieval procedures such as microwave and protease pre-treatment, were clearly not suitable for a reliable recognition of vessels by automated image analysis systems. This may be explained by the extended fixation times required for large head and neck specimens. A solution for this problem may be the use of a signal amplification system based on the horseradish peroxidase catalysed reporter deposition (CARD) of biotinylated tyramine at sites of immunoreactivity [2,3]. The CARD procedure is a highly sensitive and highly specific method for signal amplification in immunohistochemistry and in situ hybridisation $[15,17,20]$. In a recent study, CARD amplification was used to enhance the immunohistochemical signal for the fully automated microvessel counting and hot spot selection by monochromatic image analysis [1].
The aims of the present study were (1) to develop an immunohistochemical protocol based on CARD technique to enhance staining results for use in true colour image analysis of head and neck specimens, (2) to assess the reproducibility of a systematic sampling procedure to quantify vessel parameters in SCCs of the head and neck, (3) to assess if vessel parameters enabled to discriminate between metastasised and nonmetastasised tumours of the tongue.

\section{Materials and methods}

Formalin-fixed, paraffin-embedded tissue sections from resection specimens of SCCs of the tongue, which exhibited variable immunostaining intensities in the standard immunohistochemical procedure were used in several immunohistochemical experiments with CARD amplification in order to select the most suitable procedure for use in true colour image analysis.

\subsection{Patient material}

From our archives we selected 10 patients with a SCC of the mobile tongue, 6 patients with locoregional lymph node metastasis and 4 patients without metastasis during follow-up of two years or more. For each of these patients, the hematoxylin-eosin (HE) stained tissue sections of all tissue blocks obtained from the resection specimen were examined in order to select one block that contains a major part of the tumour. Tissue sections of these selected tissue blocks were immunohistochemically stained with the following CD34/CARD procedure.

\subsection{Immunohistochemistry}

\subsubsection{Standard immunohistochemical procedure}

The standard immunohistochemical procedure used in this study started with formalin-fixed, paraffinembedded tissue sections of $4 \mu \mathrm{m}$ thickness that were mounted on Superfrost Plus slides (Menzel Gläser, Braunschweig, Germany) and dried overnight at $56^{\circ} \mathrm{C}$. Subsequently, the sections were deparaffinised, treated with $1 \% \mathrm{H}_{2} \mathrm{O}_{2}$ in methanol for 20 minutes, and rinsed three times in phosphate buffered saline (PBS, $\mathrm{pH}$ 7.4) for 5 minutes. After pre-incubation with normal horse serum $100 \%$ (Vector Laboratories, Burlington, USA) for 20 minutes at room temperature (RT), an overnight incubation at $4{ }^{\circ} \mathrm{C}$ with CD34 (Qb-END, Biogenex, San Ramon, USA) $1: 2$ in PBS/1\% bovine serum al- 
bumin (PBS/1\% BSA, Sigma, St. Louis, USA) was performed, followed by three rinsing steps of $10 \mathrm{~min}-$ utes in PBS, incubation with biotinylated horse-antimouse serum (Vectastain ABC-kit, Vector Laboratories) $1: 200$ in $\mathrm{PBS} / 1 \% \mathrm{BSA}$ (Sigma) for $30 \mathrm{~min}-$ utes at RT, and three rinsing steps of 5 minutes in PBS. Subsequently, the tissue sections were incubated for 45 minutes with peroxidase (PO) labelled avidin-biotin-complex (ABC ${ }^{\mathrm{PO}}$, $\mathrm{ABC}$ Elite, 1:100; Vector Laboratories) or with alkaline phosphatase (AP) labelled avidin-biotin-complex (ABC ${ }^{\mathrm{AP}}$, ABC Standard, $1: 100$, Vector Laboratories). After three rinsing steps of 5 minutes each in PBS, peroxidase was visualised with $0.05 \%$ diaminobenzidine $/ 0.15 \% \mathrm{H}_{2} \mathrm{O}_{2}$ (DAB, Sigma) in PBS for 5 minutes and alkaline phosphatase with Vector Red (alkaline phosphatase kit I, Vector Laboratories) for 30 minutes at RT, according to the suppliers instructions. Finally, the sections were rinsed in tap water, slightly counterstained with Mayer's hematoxylin, rinsed in tap water, dehydrated, and mounted with Permount (Fisher Scientific, Fair Lawn, USA). To avoid diffusion of the enzyme precipitate of Vector Red the sections needed to be dehydrated rapidly.

\subsubsection{Biotinylation of tyramine}

One hundred mg sulfosuccinimidyl-6-(biotinimide) hexanoate (NHS-LC-biotin) (Pierce, Rockford, USA) was dissolved in $40 \mathrm{ml} 50 \mathrm{mM}$ borate buffer, $\mathrm{pH}$ 8.0. $\mathrm{Next}, 30 \mathrm{mg}$ tyramine- $\mathrm{HCl}$ (Sigma) was added. The solution was agitated overnight at RT and filtered. Before application, biotinylated tyramide (BT) was diluted in PBS, with $0.01 \% \mathrm{H}_{2} \mathrm{O}_{2}$.

\subsubsection{Signal amplification experiments}

In order to optimise the CARD procedure, tissue sections of SCCs exhibiting intra- and interspecimen variations in the intensities of immunohistochemically stained vessels were incubated with BT at different dilutions $(1: 25,1: 50,1: 100,1: 500$ and $1: 1000)$ and different BT incubation times (1, 2, 4, 8 and 12 minutes). Proceeding the CARD procedure, the standard immunohistochemical procedure was applied with the incubation time for $\mathrm{ABC}^{\mathrm{PO}}$ reduced to 30 minutes. After three rinsing steps of 5 minutes in PBS, incubations with $\mathrm{BT}$ at the aforementioned concentrations and incubation times were performed. After three rinsing steps in PBS for 5 minutes the sections were incubated with $\mathrm{ABC}^{\mathrm{PO}} 1: 100$ or $\mathrm{ABC}^{\mathrm{AP}} 1: 100$ for 20 minutes, and rinsed three times in PBS for 5 minutes. Peroxidase was visualised with DAB incubation for 5 minutes and alkaline phosphatase with Vector Red incuba- tion for 30 minutes. The processing steps that followed these DAB and Vector Red incubation were the same as described before.

\subsection{Tissue sampling}

The sections of each of the 10 SCCs of the tongue were examined at low power magnification (objective $4 \times)$ and the area of tumour tissue within the section was demarcated. The demarcated tumour tissue was manually sampled at higher magnification (objective $10 \times)$ with a systematic sampling technique. In short, neighbouring fields in a horizontal and vertical direction were sampled subsequently, thus reciprocating from left to right, starting at the top going to bottom through the tissue section. In cases with a large tumour area, two or more neighbouring fields were skipped in a horizontal direction, in order to limit the number of fields. Only fields including enough tumour tissue and tumour-stroma were included. Fields containing more than $50 \%$ of pre-existing vessel-rich structures such as striated muscle, lamina propria or peripheral nerves were skipped. Fields containing more than 50\% of necrosis or artefacts were also skipped. In this manner, the whole tumour in the tissue section was sampled with 40-60 fields. The actual size of each field was $0.17 \mathrm{~mm}^{2}$. All digitised images were stored on magneto-optical disks.

\subsection{Image analysis}

Image analysis was performed using a Vidas ${ }^{\text {plus }}$ system (Kontron $\mathrm{GmbH}$, Eching, Germany). Images were recorded by a three-chip CCD camera (DXC325P, Sony, Tokyo, Japan) mounted on a conventional light microscope (Axioskop, Zeiss, Jena, Germany) using a $10 \times$ objective (numerical aperture $=0.3$ ). At this magnification, the recorded fields measured $0.4 \times$ $0.425 \mathrm{~mm}$ (pixel size $0.78 \times 0.83 \mu \mathrm{m}^{2}$ ). Digitised images were stored on magneto-optical disc (Borsu Systema, Lelystad, The Netherlands) as true colour (24-bit RGB) images. Prior to recording images of tissue specimens, an image of an empty microscopic field and a dark-current image were stored in order to correct for unequal illumination and dark current of the camera, respectively.

Recognition of the CD34 stained objects was performed using an algorithm described elsewhere in detail [23]. In short, the well-known red-green-blue (RGB) to hue, saturation, intensity (HSI) conversion was applied to the optical densities for the three cam- 
era channels instead of the channel intensities. This hue, saturation, density (HSD) colour model was found to be superior to the existing RGB and HSI colour models for stain recognition in transmitted light microscopy [23]. The HSD model was implemented on the Vidas ${ }^{\text {plus }}$ system using the $\mathrm{C}$ free programming facilities. Application of the RGB to HSD conversion results in two chromatic co-ordinates for each image point (pixel). Due to camera limitations, the coordinates within the chromatic plane are confined to a triangular region. Each stain is represented by an ellipsoid subdomain in this chromaticity triangle. A number of test specimens, containing only pure $\mathrm{DAB}$ or Vector Red, was used to determine the locations and extensions of the subdomains of these stains in the chromaticity triangle. Recognition of vascular structures was performed as follows. Image pixels with chromatic co-ordinates inside the ellipsoid regions of the used stains were labelled as positive. Pixels with optical density (OD) smaller than 0.2 were never labelled positive, whereas pixels with OD greater than 0.7 were always labelled positive, because chromatic information of pixels originating from very low or very high amounts of stain is inaccurate. Next, 8-connected neighbouring pixels were grouped together to form objects that possibly represent vascular profiles. These binary objects were dilated twice, after which holes inside the objects were filled. Next, objects were eroded twice to restore their original size. These binary operations resulted in filling of lumina, closure of small gaps in the object contours, and slight smoothing of the object contours so that values of perimeter and diameter are more accurate. The final step consisted of removal of objects with area smaller than 50 pixels, as these were found too small to represent vascular structures.

The resulting vessel contours were projected in the original colour images, providing a facility for interactive deletion of incorrectly segmented vessel profiles, due to larger gaps in the vessel contour. Because it was not always possible to store fields that consisted entirely of vital tumour tissue, non-tumour regions could be interactively excluded from further analysis.

Finally, parameters were calculated describing the MVD and characteristics of individual vessel profiles. Only vessel profiles entirely localised within the field were used to measure individual vessel parameters, whereas the "forbidden line"-method was used to measure MVD [12]. In addition to parameters provided by the Vidas ${ }^{\text {plus }}$ system (Table 1), a measure for the diameter (DIAM) was implemented. The latter parameter was obtained as follows. The medial axis of an
Table 1

Vessel parameters and their definitions used in this study

\begin{tabular}{ll}
\hline Parameter & Description \\
\hline MVD & Number of vessels per $\mathrm{mm}^{2}$, \\
& corrected for measured area \\
AREA & Area of individual vessels \\
PERIM & Perimeter of individual vessels \\
DIAM & Diameter of individual vessels \\
\%CAT1 & Percentage of vessels with \\
& DIAMETER $<5 \mu \mathrm{m}$ \\
\%CAT2 & Percentage of vessels with \\
& $5 \mu \mathrm{m} \leqslant$ DIAMETER $<10 \mu \mathrm{m}$ \\
\%CAT3 & Percentage of vessels with \\
& $10 \mu \mathrm{m} \leqslant$ DIAMETER $<15 \mu \mathrm{m}$ \\
& Percentage of vessels with \\
\hline
\end{tabular}

object was determined using binary erosions and for each pixel on this axis, the shortest distance to the object contour was measured using a distance transform. DIAM is defined as the maximum value of the shortest distances multiplied by a factor two.

\subsection{Interobserver reproducibility}

In the aforementioned procedure, two observer dependent steps may cause interobserver variations: (1) interactive correction of stored images and (2) field selection using the systematic sampling procedure. In order to assess the interobserver reproducibility of the interactive correction step, 10 SCCs of the tongue were sampled by one observer (EH) and the stored images were independently corrected by two observers (EH and VC). Subsequently, the same 10 SCCs of the tongue were sampled and corrected independently by a second observer (VC).

\subsection{Statistics}

Statistical analysis was performed using SPSS 8.0 for Windows (SPSS Inc., Chicago, USA). Based on MVD, for each tumour the 25 most vascularised fields were selected from the 40-60 sampled fields. Averages for all vessel parameters were calculated over all vessels in the most vascularised field (henceforth called the "hot-spot") and in the 5, 10, 15, and 25 fields with highest MVDs. The vessel profiles were subdivided in 4 diameter categories, consisting of profiles with DIAM $<5,5 \leqslant$ DIAM $<10,10 \leqslant$ DIAM $<15$, and DIAM $\geqslant 15$, respectively. Subsequently, the percentage of vessels in each category was calculated for the 
hot spot and the 5, 10, 15, and 25 fields with highest MVDs.

The interobserver reproducibility of the interactive correction step and the systematic sampling procedure were assessed by a Spearman's rank correlation analysis and analysis of variance (ANOVA). In the ANOVA model, the main effects of patients and observers, as well as the interaction term between observer and patient were considered. ANOVA was also used to assess the differences between metastasised and nonmetastasised tongue carcinomas.

\section{Results}

\subsection{Immunohistochemistry}

CARD amplification with BT dilution of $1: 50$ and BT incubation time of 5 minutes was ultimately selected to visualise vessels in SCCs of the head and neck for assessment by image analysis. This protocol resulted in very intensely stained vessels and sprouting and migrating endothelial cells, whereas the tumour stroma did not exhibit background staining. In contrast, the pre-existing structures such as striated muscle cells and peripheral nerves gave high background staining. The BT dilutions of $1: 500$ and $1: 1000$ did not amplify the signal enough for cases with very weak staining intensities of vessels using the standard procedure. The $1: 25$ BT concentration did not give a substantial improvement of the signal amplification in comparison to $1: 50$. The shortest BT incubation times also resulted in insufficient signal amplification. In cases where the standard immunohistochemical procedure resulted in unstained or very weakly stained vessels the effect of CARD amplification was considerable (Figs 1a-e). In these cases, the CARD amplification step did not only result in an enhancement of the recognition of CD34 stained objects, but also in a larger number of immunohistochemically stained objects (Figs 1a,b). This was found in tissue sections in which the colour was developed by incubating with DAB as well as Vector Red (Figs 1b,c). In tumours in which the vessels were more adequately stained by the standard immunohistochemical protocol, CARD amplification gave more intensely stained vessels, but the differences in the number of stained objects were less obvious (Figs 1d,e).

In the DAB stained tissue sections, the contours of the vessel profiles were more sharply demarcated than in the Vector Red stained tissue sections (Figs 1b,c). In the Vector Red stained sections the boundaries of the vessels were not only more blurred, but exhibited also more interspecimen variation in blurring than the DAB stained sections. Based on these differences in staining characteristics of DAB and Vector Red, the former was selected for the use in true colour image analysis. In Fig. 1f, the results of the automatic recognition of DAB stained vessels, without interactive corrections, are given. Automatic recognition of vessels may be seriously hampered in regions (especially in striated muscles) with high background staining. The very elongated vessel transsections in the stroma of the tumour are sometimes erroneously segmented by the image analysis system as a string of multiple small vessels.

\subsection{Reproducibility of tissue sampling}

Correlation analysis revealed that the overall interobserver reproducibility of the interactive correction step was good with intercorrector correlation $r 1 \geqslant$ $0.75(p \leqslant 0.01)$ for the MVD and size parameters in the systematically determined hot-spot (Table 2). The percentages of vessels in the four categories were less reproducible, especially for \%CAT 1 and \%CAT4. The intercorrector correlation coefficients increased when vessel parameters were averaged over an increasing number of fields (Table 2). Averaging parameters from vessels in the ten highest vascularised fields resulted in $r 1$-values $>0.96$ and in $p$-values $<0.001$ for all parameters with exception of \%CAT1. Addition of more fields in the averaging did not give a substantial improvement of the reproducibility of the interactive correction step (Table 2).

Correlation analysis disclosed that the overall interobserver reproducibility of systematic determination of the hot spot is poor with low and even negative values for the interobserver correlation $(r 2)$ for all the vessel parameters (Table 2). Using vessel parameters obtained from averaging all vessels in the five highest vascularised fields found by systematic tissue sampling resulted in a substantial improvement of the interobserver reproducibility of the sampling procedure $(r 2 \geqslant 0.64, p \leqslant 0.05)$ for all parameters with exception of \%CAT1 and \%CAT4. Using the mean values obtained from the ten most vascularised fields resulted in acceptable interobserver reproducibility of all vessel parameters with exception of \%CAT1 and \%CAT4, with interobserver correlation coefficients $r 2 \geqslant 0.76$ and $p$-values $\leqslant 0.01$ (Table 2). Averaging vessel parameters over more than 10 fields gave no substantial improvement of the interobserver reproducibility of the 

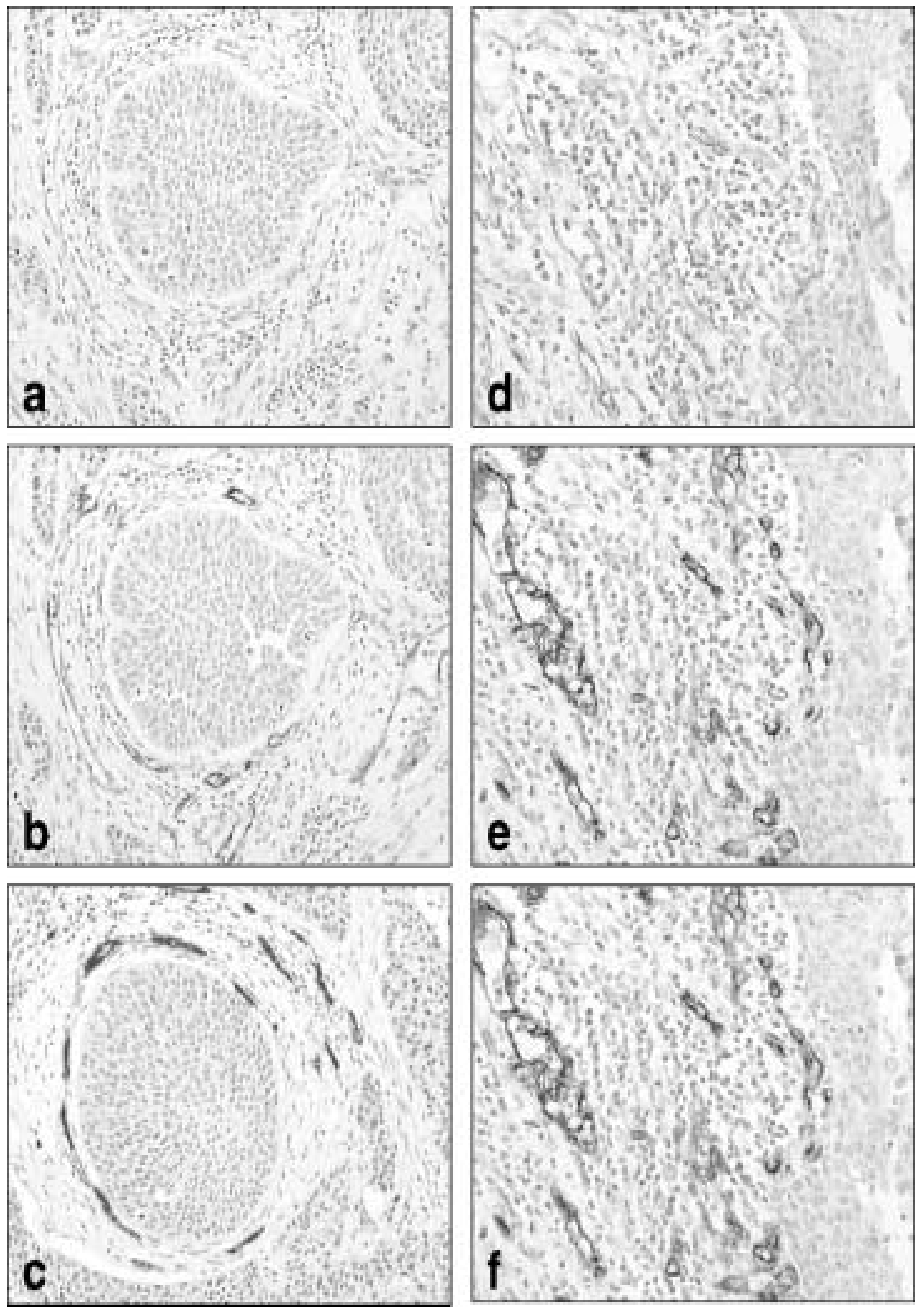

Fig. 1. Immunohistochemical staining results for the standard procedure and in combination with CARD amplification. An immunohistochemically stained tisue section of tongue carcinoma, with very weakly stained and even unstained vessels using the standard procedure with DAB (a) and the successive, serial section using CARD amplification, disclosing more vessel profiles and more intensely stained vessels with DAB (b). A non-successive, serial section of the same area in the same tumour, with vessels visualised with Vector Red after CARD amplification also showed intensely stained vessels, however Vector Red gave more blurred vessel contours than DAB (c). Another SCC with stained vessels using the standard procedure with DAB (d) and the successive, serial section using CARD amplification exhibiting more intensely stained vessels, no background staining, and plasma cells of the inflammatory infiltrate remained unstained (e). The same image as (e), with vessel profiles found after automated recognition by the image analysis system are marked with green contours (f). This figure can be viewed on http://www.esacp. org/acp/2001/22-4/hannen.htm. 
Table 2

The interobserver correlations for the correction step $(r 1)$ and for the systematic sampling $(r 2)$ and their two-tailed $p$-values are given for the MVD and other parameters in the hot spot and averaged over the 5, 10, 15, and 25 highest vascularised fields, determined by systematic tissue sampling

\begin{tabular}{|c|c|c|c|c|c|c|c|c|c|c|c|}
\hline & & \multicolumn{2}{|c|}{ Hot spot } & \multicolumn{2}{|c|}{ Top 5 fields } & \multicolumn{2}{|c|}{ Top 10 fields } & \multicolumn{2}{|c|}{ Top 15 fields } & \multicolumn{2}{|c|}{ Top 25 fields } \\
\hline & & $r$ & $p$ & $r$ & $p$ & $r$ & $p$ & $r$ & $p$ & $r$ & $p$ \\
\hline \multirow[t]{2}{*}{ MVD } & $r 1$ & 0.84 & 0.002 & 0.97 & $<0.001$ & 0.98 & $<0.001$ & 0.99 & $<0.001$ & 0.99 & $<0.001$ \\
\hline & $r 2$ & 0.48 & n.s. & 0.64 & 0.045 & 0.77 & 0.009 & 0.84 & 0.003 & 0.91 & $<0.001$ \\
\hline \multirow[t]{2}{*}{ AREA } & $r 1$ & 0.81 & 0.005 & 0.95 & $<0.001$ & 0.99 & $<0.001$ & 0.99 & $<0.001$ & 0.99 & $<0.001$ \\
\hline & $r 2$ & 0.37 & n.s. & 0.70 & 0.026 & 0.76 & 0.010 & 0.81 & 0.004 & 0.83 & 0.003 \\
\hline \multirow[t]{2}{*}{ PERIM } & $r 1$ & 0.75 & 0.012 & 0.96 & $<0.001$ & 0.99 & $<0.001$ & 0.99 & $<0.001$ & 0.99 & $<0.001$ \\
\hline & $r 2$ & 0.57 & n.s. & 0.69 & 0.029 & 0.79 & 0.006 & 0.83 & 0.003 & 0.78 & 0.008 \\
\hline \multirow[t]{2}{*}{ DIAM } & $r 1$ & 0.82 & 0.004 & 0.96 & $<0.001$ & 0.99 & $<0.001$ & 0.98 & $<0.001$ & 0.99 & $<0.001$ \\
\hline & $r 2$ & 0.50 & n.s. & 0.69 & 0.028 & 0.77 & 0.009 & 0.78 & 0.007 & 0.83 & 0.003 \\
\hline \multirow[t]{2}{*}{ \%CAT1 } & $r 1$ & 0.63 & n.s. & 0.83 & 0.003 & 0.87 & 0.001 & 0.95 & $<0.001$ & 0.93 & $<0.001$ \\
\hline & $r 2$ & -0.10 & n.s. & 0.12 & n.s. & 0.35 & n.s. & 0.45 & n.s. & 0.39 & n.s. \\
\hline \multirow[t]{2}{*}{$\%$ CAT2 } & $r 1$ & 0.69 & 0.029 & 0.94 & $<0.001$ & 0.96 & $<0.001$ & 0.96 & $<0.001$ & 0.99 & $<0.001$ \\
\hline & $r 2$ & 0.02 & n.s. & 0.88 & 0.001 & 0.90 & $<0.001$ & 0.92 & $<0.001$ & 0.90 & $<0.001$ \\
\hline \multirow[t]{2}{*}{ \% CAT3 } & $r 1$ & 0.67 & 0.036 & 0.92 & $<0.001$ & 0.98 & $<0.001$ & 0.97 & $<0.001$ & 0.99 & $<0.001$ \\
\hline & $r 2$ & 0.47 & n.s. & 0.89 & 0.001 & 0.92 & $<0.001$ & 0.88 & 0.001 & 0.84 & 0.002 \\
\hline \multirow[t]{2}{*}{ \%CAT4 } & $r 1$ & 0.28 & n.s. & 0.97 & $<0.001$ & 0.99 & $<0.001$ & 0.99 & $<0.001$ & 0.99 & $<0.001$ \\
\hline & $r 2$ & -0.41 & n.s. & 0.29 & n.s. & 0.55 & n.s. & 0.66 & 0.044 & 0.82 & 0.004 \\
\hline
\end{tabular}

systematic sampling procedure. Averaging vessel parameters over the ten most vascularised fields found by systematic tissue sampling was found to result in an acceptable overall interobserver reproducibility in SCCs of the tongue.

For the interactive correction step, ANOVA calculated over data from the 10 most vascularised fields disclosed no significant interactions between observers and patients. A significant interaction would indicate that the interobserver reproducibility varies between patients. The effect of interactive correction was not significant for any parameter, except for the MVD $(p=0.01)$. However, variations between patients were found highly significant for almost all parameters. The effect of field sampling by two independent observers showed a significant interaction between observer and patient for a number of parameters (AREA, DIAM, and MVD), indicating a significant sampling effect for some patients for these parameters. This is shown graphically in Fig. 2 for MVD and DIAM. For PERIM and \%CAT3, a significant interobserver effect was found ( $p=0.001$ and $p=0.02$, respectively).

\subsection{Metastasised versus non-metastasised tumours}

Using the results obtained from the 10 most vascularised test fields, the mean values and standard deviations of the parameter \%CAT1 were $20.4 \pm 3.0$ and
$16.7 \pm 4.1$ for non-metastasised and metastasised tumours, respectively. ANOVA disclosed that this difference is statistically significant $(p=0.02)$. For the other parameters, no significant differences between these two groups were found.

\section{Discussion}

Figures on http://www.esacp.org/acp/2001/22-4/ hannen.htm.

The results of the present study show the developed immunohistochemical protocol based on CARD signal amplification enhanced the staining results. The sensitivity, specificity, and signal-to-noise ratio of the immunohistochemical detection of blood vessels with the monoclonal antibody directed against CD34 were improved. The combination of high intensity of the specific immunohistochemical signals and the absence of background staining is an important condition for successful automated recognition of immunohistochemically stained objects in tissue sections by image analysis systems. Background staining appeared to be a major limiting factor in the automated recognition of vessel profiles using true colour image analysis [24]. In a recent study on angiogenesis in breast cancer, application of CARD amplification proved necessary for the automatic recognition of vessels by monochromatic 


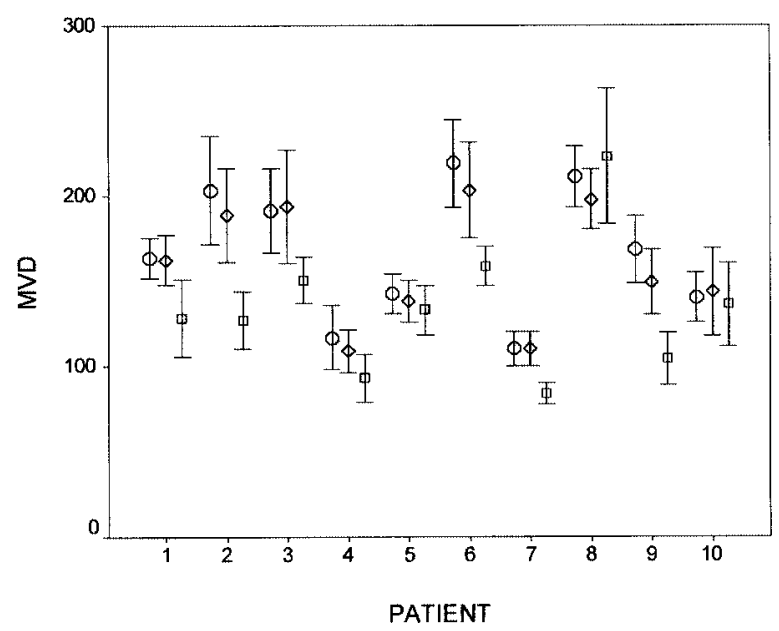

(a)

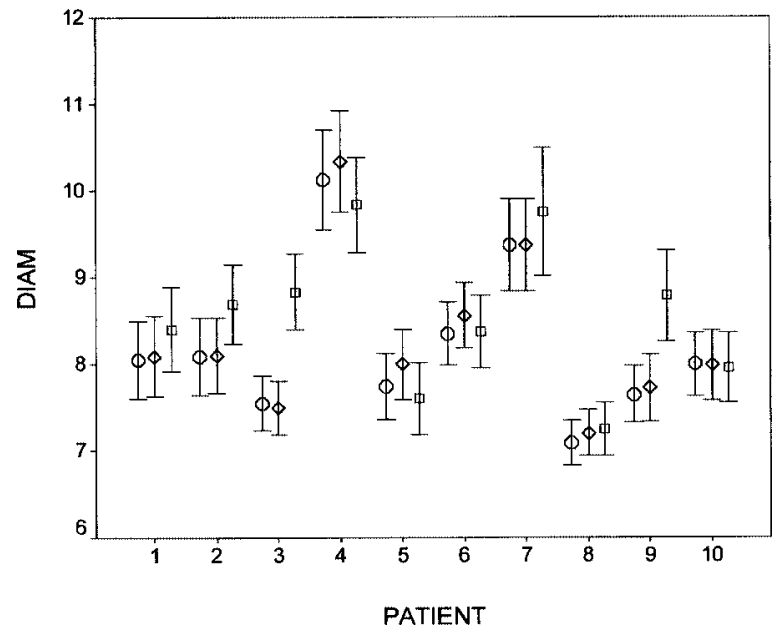

(b)

Fig. 2. Interobserver reproducibility for the interactive correction step and the systematic sampling of microvessel density and vessel diameter for 10 patients with a tongue carcinoma. For each patient, the mean values and 95\% confidence intervals of the microvessel density (MVD) (a) and DIAM (b) obtained from the 10 highest vascularised fields found by sampler 1 and corrector $1(\circ)$, sampler 1 and corrector $2(\diamond)$, sampler 2 and corrector $2(\square)$.

image analysis using the monoclonal antibody directed against CD31 [1]. Antibodies directed against CD31 have been proposed as standard for the visualisation of vessels [25]. However, it cannot be recommended for use in the automatic recognition of microvessels in chronically inflamed tumours, because plasma cells are also stained by CD31. This problem was encountered in the aforementioned study on breast carcinomas and required an interactive correction step to eliminate the falsely recognised plasma cells [1].

The Chalkley point counting procedure has been proposed as the standard method for quantification of intratumour MVD due to inadequate reproducibility of the automated recognition of vessel profiles in tissue sections due to the heterogeneity of microvessel morphology, e.g., size, length and anastomoses, and local differences in immunostaining intensity [25]. In the present study it was shown that problems with regard to the intra- and interspecimen variations of the immunohistochemical signal were considerably reduced by combination of the CARD amplification and true colour image analysis using the HSD colour model. The CARD procedure resulted in more uniform and enhanced staining intensities and sharply demarcated vessel boundaries when DAB was used. Because in the HSD model colour information is used for recognition of vessels, intra- and interspecimen variations in staining density had less effect on the segmentation of vessel profiles than when the widely used RGB or HSI models were applied. The advantages of the use of the HSD colour model in transmitted light microscopy were recently reported [23]. In the present study, it was shown that interactive correction of stored images by two independent observers resulted in a good interobserver reproducibility. Only for the MVD, a weakly significant corrector effect was observed, probably reflecting the difficulty in defining criteria for individual vessel profiles. However, this problem hampers any procedure for quantification of the MVD.

Reliable automated recognition of immunohistochemically stained vessels has important advantages in comparison to point-counting procedures, such as the recommended Chalkley method. Size and shape parameters can be used to subclassify blood vessels, enabling assessment of the relationship between subclasses of vessels and biological behaviour of tumours. In a recent study on human melanoma xenografts with different metastatic behaviour, no significant differences between the two melanomas were found for immunohistochemically stained objects with diameter $<3 \mu \mathrm{m}$ and diameter $>12 \mu \mathrm{m}$, whereas the most significant differences were found for vessels with a diameter in the range of 6-9 $\mu \mathrm{m}$ [24]. The results of the latter study demonstrated the need to determine subgroups of microvessels preferentially used in the complex process of metastasis. In the present study on a very small series of patients with a tongue carcinoma, it was shown that significant differences between metastasised and non-metastasised tumours were found for the percentages of vessels with a di- 
ameter smaller than $5 \mu \mathrm{m}$, whereas the MVD did not differ significantly.

The systematic sampling procedure for SCCs of the tongue with 40-60 fields is unsuitable for a reliable hot spot selection in tissue sections, as was shown in the present study. Vessel parameters obtained from the field with the highest vessel density (i.e., systematically determined hot spot) were not reproducible. A substantial improvement of the interobserver correlation was obtained by averaging these vessel parameters over a larger numbers of the most vascularised fields. Calculation of the moving average of these parameters revealed that the mean values obtained from the 10 most vascularised fields, found by systematic sampling, resulted in an acceptable overall interobserver reproducibility. However, the interobserver reproducibility of field sampling varied between patients, and the differences between the two observers were significant for some patients even after averaging 10 or more of the highest vascularised fields. The interobserver reproducibility of systematic tissue sampling is determined by both intratumour variations due to tumour heterogeneity and by the sampled tumour area, which is determined by the number of systematically sampled fields. Other studies using complete sampling of the whole tumour area in tissue sections disclosed that this procedure resulted in highly reproducible hot spot selection $[1,24]$. However, it remains to be elucidated whether the MVD in the hot spot, found by complete sampling of the tumour area in one single tissue section, is more representative for the whole tumour than the MVD estimated by the mean value of the 10 most vascularised fields found by a systematic sampling procedure in one tissue section. De Jong et al. [5] found a noteworthy heterogeneity in microvessel counts in breast tumours between different areas from the same section. This is in agreement with our observations in SCCs of the tongue. Heterogeneity in microvessel counts was also found between corresponding areas in different sections from the same tissue block, and between blocks from the same tumour. Differences between blocks caused the larger part of the total variance of microvessel counts [5]. It is likely that the inter-block variations also cause a major part of the total variance in other tumour types, including squamous cell carcinomas of the tongue.

In the literature, there is still no consensus concerning the number of highest vascularised fields in tumours that must be used to obtain a reliable and reproducible estimation of the intratumoural MVD [26]. In a number of studies, the MVD in only one hot spot was used [19,27]; other investigators used the mean value of the three or four most vascularised spots in a tumour section $[7,14,18]$. It is questionable whether there is an unequivocal answer to this question, because the ideal number of "hot spots" needed for a reproducible estimation of the MVD representative for the whole tumour may depend on tumour type and on the tissue sampling procedure. In our opinion, for each tumour type the number of highest vascularised areas ("hot spots") that is required to obtain a reproducible estimation of intratumoural MVD must be determined by moving average experiments.

We conclude that CARD amplification is needed for reliable recognition of vessels by automated image analysis of head and neck tissue samples. Tumour heterogeneity is a limiting factor for the application of systematic tissue sampling, but this is true for all other procedures in which hot spot selection is based on a single tissue section or on a biopsy specimen. Especially, when vessel parameters will be used to construct a discriminant function to predict the biological behaviour of a tumour for individual patients, one must be aware of sampling errors that may cause misclassifications.

\section{Acknowledgements}

Contract grant sponsor: Dutch Cancer Society, grant number NUKC-NKB-KWF-94-726. The authors thank Prof. Dr. D.J. Ruiter for the valuable discussions. In addition, we thank Ms. J.H. Gemmink for the immunohistochemical staining.

\section{References}

[1] J.A.M. Beliën, S. Somi, J.S. De Jong, P.J. Van Diest and J.P.A. Baak, Fully automated microvessel counting and hot spot selection by image processing of whole tumor sections in invasive breast cancer, J. Clin. Pathol. 52 (1999), 184-192.

[2] M.N. Bobrow, T.D. Harris, K.J. Shaughnessy and G.J. Litt, Catalyzed reporter deposition, a novel method of signal amplification. Application to immunoassays, J. Immunol. Methods $\mathbf{1 2 5}$ (1989), 279-285.

[3] M.N. Bobrow, K.J. Shaughnessy and G.J. Litt, Catalyzed reporter deposition, a novel method of signal amplification. II. Application to membrane immunoassays, J. Immunol. Methods 137 (1991), 103-112.

[4] W.C. Cochran, Sampling Techniques, Wiley and Sons, New York, 1977.

[5] J.S. De Jong, P.J. Van Diest and J.P.A. Baak, Heterogeneity and reproducibility of microvessel counts in breast cancer, $L a b$. Invest. 73 (1995), 922-926. 
[6] S.B. Fox, Tumor angiogenesis and prognosis, Histopathology 30 (1997), 294-301.

[7] S.B. Fox, R.D. Leek, M.P. Weekes, R.M. Whitehouse, K.C. Gatter and A.L. Harris, Quantitation and prognostic value of breast cancer angiogenesis: comparison of microvessel density, Chalkley count and computer image analysis, J. Pathol. 177 (1995), 275-283.

[8] G. Gasparini, N. Weidner, S. Maluta, R. Pozza, P. Boracchi, M. Mezzetti, A. Testolin and P. Bevilacqua, Intratumoral microvesseldensity and p53 protein: correlation with metastasis in head-and-neck squamous-cell carcinoma, Int. J. Cancer $\mathbf{5 5}$ (1993), 739-744.

[9] L.L. Gleich, P.W. Biddinger, F.D. Duperier and J.L. Gluckman, Tumor angiogenesis as a prognostic indicator in $\mathrm{T} 2-\mathrm{T} 4$ oral cavity squamous cell carcinoma: a clinical-pathologic correlation, Head Neck 19 (1997), 276-280.

[10] R.C. Gonzalez and R.E. Woods, Digital Image Processing, Addison-Wesley Publishing Company, Reading, MA, 1992.

[11] H.J.G. Gundersen and E.B. Jensen, The efficiency of systematic sampling in stereology and its prediction, J. Microsc. 147 (1987), 229-263.

[12] H.J.G. Gundersen, Notes on the estimation of the numerical density of arbitrary profiles: The edge effect, J. Microsc. 111 (1977), 219-223.

[13] S. Hansen, D.A. Grabau, C. Rose, M. Bak and F.B. Sørensen, Angiogenesis in breast cancer: a comparative study of the observer variability of methods for determining microvessel density, Lab. Invest. 78 (1998), 1663-1673.

[14] E.R. Horak, R. Leek, N. Klenk, S. LeJeune, K. Smith, N. Stuart, M. Greenall, K. Stepniewska and A.L. Harris, Angiogenesis, assessed by platelet/endothelial cell adhesion molecule antibodies, as indicator of node metastases and survival in breast cancer, Lancet 340 (1992), 1120-1124.

[15] H.M.J. Kerstens, P.J. Poddighe and A.G.J.M. Hanselaar, A novel in situ hybridization signal amplification method based on the deposition of biotinylated tyramine, J. Histochem. Cytochem. 43 (1995), 347-352.

[16] D.A. Leedy, D.R. Trune, J.D. Kronz, N. Weidner and J.I. Cohen, Tumor angiogenesis, the p53 antigen, and cervical metastasis in squamous cell carcinoma of the tongue, Otolaryngol. Head Neck Surg. 111 (1994), 417-422.

[17] G. King, S. Payne, F. Walker and G.I. Murray, A highly sensitive detection method for immunohistochemistry using biotinylated tyramide, J. Pathol. 183 (1997), 237-241.

[18] P.D. Kohlberger, A. Obermair, G. Sliutz, H. Heinzl, H. Koelbl, G. Breitenecker, G. Gitsch and C. Kainz, Quantitative immunohistochemistry of Factor VIII-related antigenin breast carci- noma. A comparison of computer-assisted image analysis with established counting methods, Am. J. Clin. Pathol. 105 (1996), 705-710.

[19] S.P. Leon, R.D. Folkerth and P.M. Black, Microvessel density is a prognostic indicator for patients with astroglial brain tumors, Cancer 77 (1996), 362-372.

[20] A.K. Raap, M.C.P. Van de Carput, R.A.W. Vervenne, R.P.M. Gijlswijk, H.J. Tanke and J. Wiegant, Ultra-sensitive FISH using peroxidase-mediated deposition of biotin- or fluorochrome tyramides, Hum. Mol. Gen. 4 (1995), 529-534.

[21] T. Shpitzer, M. Chaimoff, R. Gal, Y. Stern, R. Feinmesser and K. Segal, Tumor angiogenesis as a prognostic factor in early oral tongue cancer, Arch. Otolaryngol. Head Neck Surg. 122 (1996), 865-868.

[22] S.R. Tahan and A.L. Stein, Angiogenesis in invasive squamous cell carcinoma of the lip: tumor vascularity is not an indicator of metastatic risk, J. Cutan. Pathol. 22 (1995), 236-240.

[23] J.A.W.M. Van der Laak, M.M.M. Pahlplatz, A.G.J.M. Hanselaar and P.C.M. De Wilde, Hue-Saturation-Density (HSD) model for stain recognition in digital images from transmitted light microscopy, Cytometry 39 (2000), 275-284.

[24] J.A.W.M. Van der Laak, J.R. Westphal, L.J.M. Schalkwijk, M.M.M. Pahlplatz, D.J. Ruiter, R.M.W. De Waal and P.C.M. De Wilde, An improved procedure to quantify tumor vascularity using true color image analysis. Comparison with the manual hot-spot procedure in a human melanoma xenograft model, J. Pathol. 184 (1998), 136-143.

[25] P.B. Vermeulen, G. Gasparini, S.B. Fox, M. Toi, L. Martin, P. McCulloch, F. Pezzella, G. Viale, N. Weidner, A.L. Harris and L.Y. Dirix, Quantification of angiogenesis in solid human tumors: an international consensus on the methodology and criteria of evaluation, Eur. J. Cancer. 32A (1996), 2474-2484.

[26] E.R. Weibel, Stereological Methods, Vol. 1, Practical Methods for Biological Morphometry, Academic Press, London, 1979.

[27] N. Weidner, J.P. Semple, W.R. Welch and J. Folkman, Tumor angiogenesis and metastasis - correlation in invasive breast carcinoma, N. Engl. J. Med. 324 (1991), 1-8.

[28] P. Wesseling, J.A.W.M. Van der Laak, H. De Leeuw, D.J. Ruiter and P.C. Burger, Quantitative immunohistochemical analysis of the microvasculature in untreated human glioblastoma multiforme, J. Neurosurg. 81 (1994), 902-909.

[29] U.K. Zätterström, E. Brun, R. Willén, E. Kjellén and J. Wennerberg, Tumor angiogenesis and prognosis in squamous cell carcinoma of the head and neck, Head Neck 17 (1995), 312318. 


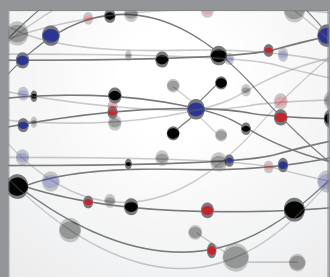

The Scientific World Journal
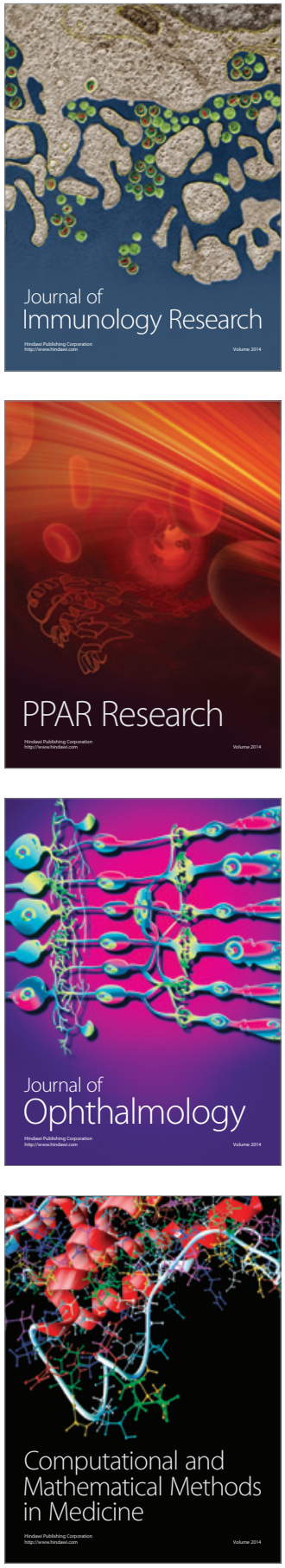

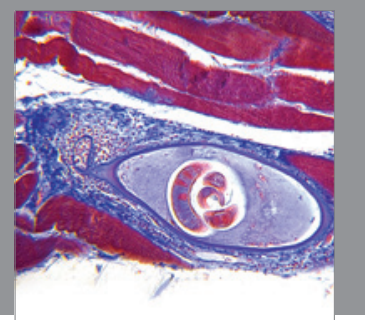

Gastroenterology

Research and Practice
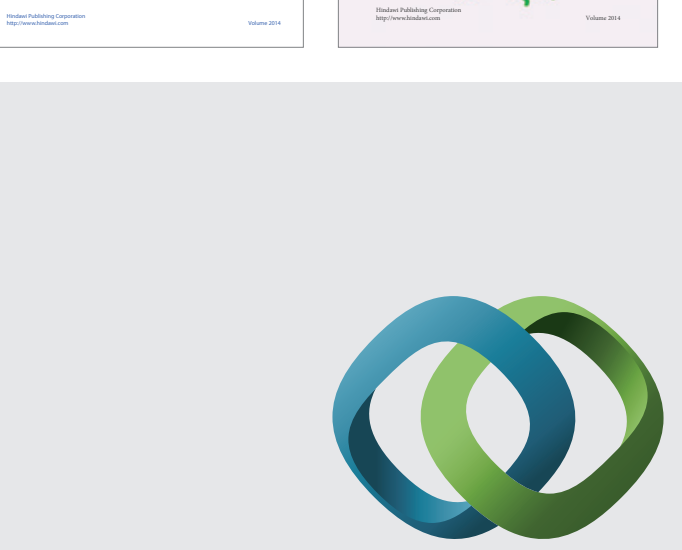

\section{Hindawi}

Submit your manuscripts at

http://www.hindawi.com
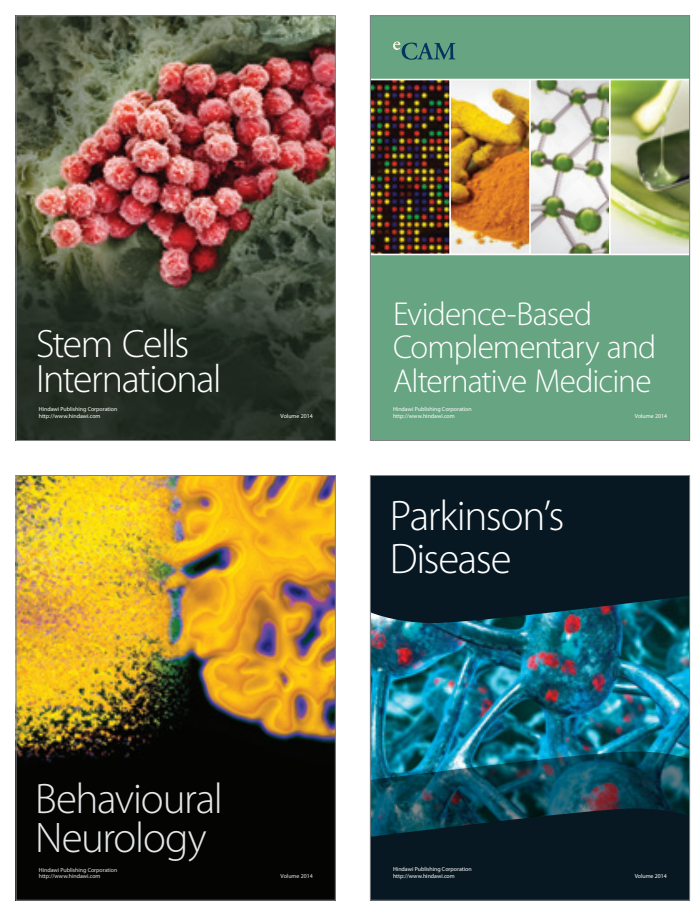

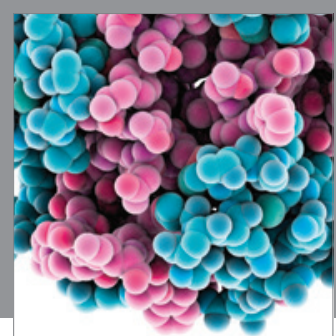

Journal of
Diabetes Research

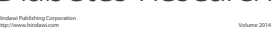

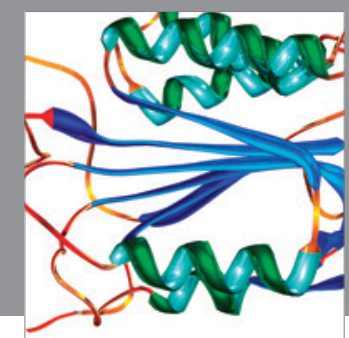

Disease Markers
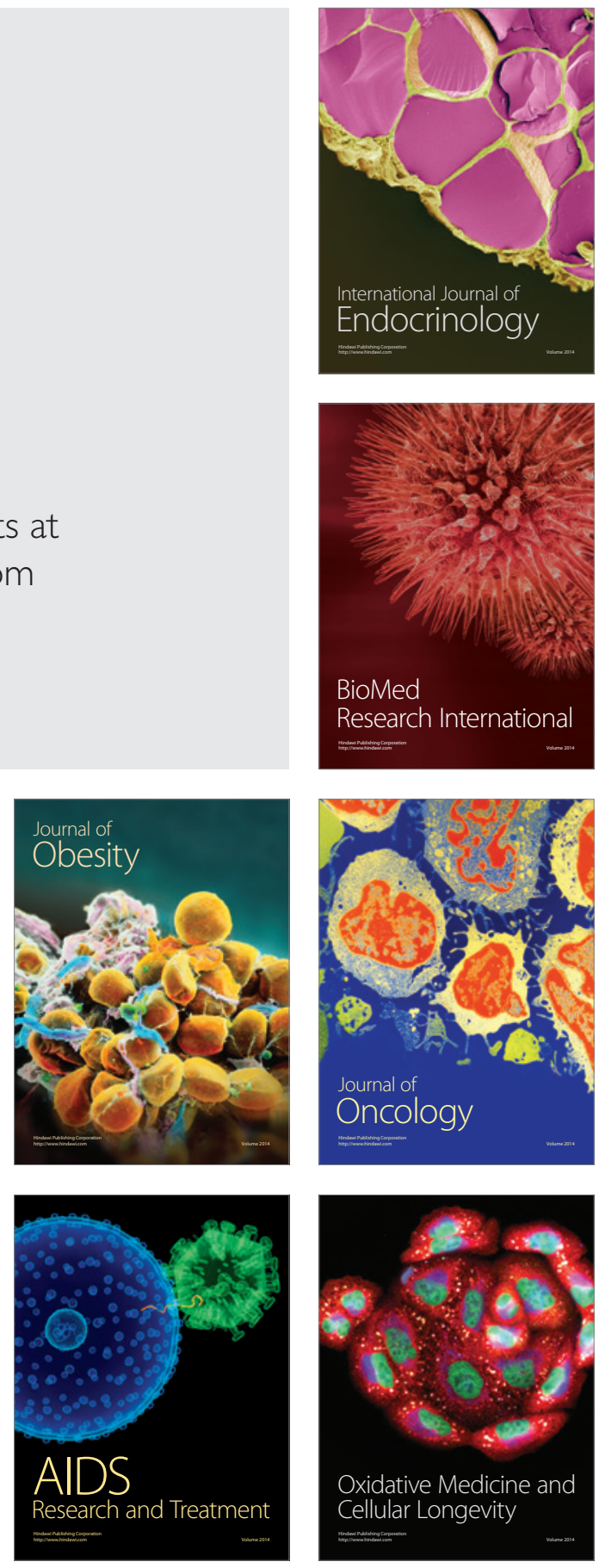